\section{AKADEMOS}

Órgano de difusión de la Red Docencia-Investigación

ISSN: $1995-4743$

Año 14 Vol. 1, n. 34 Enero-Junio 2020

AKADEMOS es una revista semestral. De amplio espacio editorial, para la publicación de trabajos inéditos de investigación, artículos de análisis, reseñas y opinión, en los distintos tópicos de las ciencias, la tecnología, las artes y la cultura.

\title{
Aportes para la renovación del canon literario escolar de educación media de El Salvador
}

\section{Contributions to the renewal of El Salvador's high school literary canon}

Mario Zetino

Licenciado en Letras. Investigador en estudios literarios y educación del Centro de Investigaciones en Ciencias y Humanidades (CICH), Universidad Dr. José Matías Delgado

mesermenoz@ujmd.edu.sv

\section{Resumen}

Este artículo busca dar aportes para la renovación del canon literario escolar de educación media de El Salvador, tanto en estructura como en prácticas, de modo que su utilización en el proceso de enseñanza aprendizaje posibilite aprendizajes significativos. La revisión de la literatura muestra que son muy pocos los estudios sobre el canon literario escolar salvadoreño. Sin embargo, los estudios existentes hacen aportes clave, como la identificación de falencias de coherencia pedagógica y de pertinencia cultural en los programas de estudio de Lenguaje y Literatura y la caracterización del modelo de enseñanza de la lite- ratura en El Salvador y del canon literario escolar de educación media como historicistas, europeizantes, masculinos y conservadores. Además, se constata que los docentes no están formados para desarrollar un proceso de enseñanza-aprendizaje en literatura enfocado en el desarrollo de competencias, sino que la enseñanza en este campo sigue un esquema bancario. Ante estos problemas, los estudios señalan las necesidades de enseñar literatura a partir de una teoría definida y explícita de la lectura literaria y de perfilar pedagógica y epistemológicamente un modelo integral de lectura literaria que considere tanto su aspecto individual como su aspecto de socialización. Por su capacidad de responder a estas necesidades, la educación literaria se plantea como 
un modelo más funcional para la enseñanza de la literatura. A partir de este marco conceptual, se formulan recomendaciones para una renovación funcional del canon literario escolar de educación media, y en relación con estas se plantean recomendaciones para la optimización del uso del canon en la educación durante la pandemia de COVID-19. Se enfatiza en la creación de un equipo multidisciplinario y de un conjunto de criterios definidos, explícitos y derivados de un modelo funcional de enseñanza de la literatura como condiciones para una reelaboración pedagógicamente efectiva del canon. Se estima que, en general, el marco teórico y las recomendaciones de este artículo son extrapolables al tercer ciclo de educación básica, por las similitudes de su canon con el de educación media.

Palabras clave: canon literario escolar, enseñanz̧a de la literatura, educación literaria, COVID-19

\section{Abstract}

This paper seeks to provide contributions to the renewal of El Salvador's high school (grades 10-11) literary canon, both in structure and in practice, so that its use in the teachinglearning process makes possible significant learning. The review of the literature shows that there are very few studies on the Salvadoran school literary canon. However, the existing studies make key contributions, such as the identification of deficiencies in pedagogical coherence and cultural relevance in the curricula of Language and Literature (the equivalent to Spanish), and the characterization of the teaching model of literature in El Salvador and its high school literary canon as histori- cist, Europeanist, masculine and conservative. Besides, it is verified that teachers are not trained to develop a teaching-learning process in literature focused on the development of skills, but that teaching in this field follows a banking scheme. Given these problems, the studies point out the needs to teach literature from a defined and explicit theory of literary reading, and to outline pedagogically and epistemologically an integral model of literary reading that considers both its individual and socialization aspects. Because of its potential to meet these needs, literary education is proposed as a more functional model for teaching literature. Based on this framework, recommendations are made for a functional renewal of the high school literary canon, and linked to these, also recommendations to optimize the use of the canon in education during the COVID-19 pandemic. Emphasis is placed on the creation of a multidisciplinary team and a set of defined, explicit criteria derived from a functional model of teaching literature, as conditions for a pedagogically effective reelaboration of the canon. It is considered that, in general, the theoretical framework and the recommendations of this article can be extrapolated to El Salvador middle school level (grades 7-9), because of the similarities of its canon with that of high school.

Key words: school literary canon, teaching of Literature, literary education, COVID-19

\section{Introducción}

La literatura, como forma de arte que es, juega un papel fundamental en la formación humana, ya que su experiencia contribuye al desarrollo de las dimensiones cognitiva, afectiva 
y cultural de las personas. En la educación formal, la enseñanza de la literatura se realiza a través de un elemento específico, que es el canon literario escolar, el conjunto de textos que se leen con diversos propósitos educativos. Sin embargo, en El Salvador, dicho potencial formativo de la literatura en muchos casos no es comprendido en el ámbito educativo, y esto ha dado como resultado un canon que, en general, resulta infuncional para el desarrollo de aprendizajes significativos, es decir que le aporten al estudiante "conocimientos y habilidades que sean valiosos por sí mismos y que sean transferibles" a la vida (Barkley \& Major, citadas en Calderón, 2020, p. 35). Esta afirmación es constatada por abundantes historias de estudiantes, padres de familia y ciudadanos en general; por las bajas notas de la PAES (Prueba de Aprendizaje y Aptitudes para Egresados de Educación Media) en el área de Literatura, y por el bajo nivel de competencias de lectura y de alfabetización cultural de la ciudadanía en general. Para asegurar una formación de calidad en el campo de la literatura, es necesario examinar y modificar la estructura del canon literario escolar y las prácticas que se desarrollan a partir de este. El presente artículo busca dar aportes para la renovación del canon, tanto en estructura como en prácticas. Específicamente, se estudia el canon de educación media o bachillerato, por ser este el nivel educativo en el que existen estudios previos sobre el tema, para poder darles continuidad y profundizarlos.

\section{Revisión de la literatura}

En El Salvador existen pocos estudios sobre el canon literario escolar y la lectura de literatura, siendo el principal referente en estos
Aguilar (2012, 2013 y 2016), cuyos aportes son fundamentales y que exponemos a continuación.

Aguilar, al introducir las conclusiones de su tesis doctoral, El papel de la literatura en el desarrollo del pensamiento crítico de las y los estudiantes de educación media: El caso salvadoreño, afirma: "La enseñanza de la literatura en la escuela secundaria necesita ser repensada" (2012, p. 329), palabras que deben ser tomadas en cuenta con urgencia, al ser el cierre de un estudio riguroso, que aporta marcos conceptuales sólidos y datos e instrumentos empíricos para evaluar la enseñanza de la literatura en el país.

\section{Noción previa del canon literario escolar salvadoreño}

Actualmente los conceptos e implicaciones del canon literario (es decir el canon general, los grandes libros de una cultura) y del canon literario escolar están siendo discutidos y debatidos en todo el mundo. Aguilar (2012, pp. 53-68) expone y problematiza estos conceptos y da un panorama de dichos debates. De este modo, muestra que el canon literario escolar no es un elemento estático ni simple, que no es sólo una lista de libros que se leen en la escuela, sino un elemento pedagógico que forma parte de una compleja red de relaciones sociales, culturales e ideológicas. Y plantea que "el modelo didáctico-pedagógico [de la literatura en El Salvador], opta por los principios que rigen la enseñanza de la literatura desde el canon clásico[,] con sus correspondientes características: excluyente, masculino y marcadamente occidental" (p. 64). Esta caracterización se desarrolla con más detalle en el apartado 3. 


\section{Panorama general: el modelo de enseñanza de la literatura en EI Salvador}

En su análisis del modelo de enseñanza de la literatura en El Salvador, Aguilar (2012) señala que, en el nivel teórico de dicho modelo, el enfoque comunicativo, que está extendido en la enseñanza de la literatura en América Latina y que orienta la enseñanza de la literatura en el país, "ha conducido a una visión reduccionista del campo literario y a una lectura fragmentaria de los textos" (p. 328). "Los indicadores de logro no son del todo satisfactorios" (p. 328). También señala que "Otra debilidad consiste en la carencia de estudios empíricos que refuercen la tesis sostenida por los defensores del enfoque comunicativo en el sentido [de] que éste representa la mejor propuesta de enseñanza de la literatura" (pp. 328-329). Por estos motivos, plantea que es preciso "hacer una relectura de los postulados teóricos tanto pedagógicos como disciplinares que sustentan dicho modelo" (p. 328).

Además, el autor analiza la actualización de programas de estudio que va del modelo de enseñanza del enfoque comunicativo al del enfoque basado en competencias (en El Salvador esto corresponde a la reforma educativa de 2008), pero "lo cierto es que los cambios no tocan la columna vertebral de aquél [del modelo de enseñanza,] ya que la literatura sigue constreñida por un patrón historicista de enseñanza y por una modalidad de bloques que reduce importantemente su potencial pedagógico" (Aguilar, 2012, p. 329).

Como un desarrollo de estos planteamientos, Aguilar (2016) analiza el concepto de lectura literaria en el marco de los procesos de enseñanza de la literatura, como condición "para el mejor aprovechamiento de las potencialidades formativas de la literatura" (p. 81). En su análisis, el autor encuentra vacíos en la Didáctica de la Lengua y la Literatura, el modelo didáctico-pedagógico en el que se basa la formación docente y la enseñanza de la literatura en El Salvador:

[...] en el campo de la de la Didáctica de la Lengua y la Literatura (DLL), pese al marcado carácter constructivista que se asume, el estudio del fenómeno de la lectura literaria ha ocupado una atención marginal. Ciertamente, a pesar del énfasis que hace la DLL en el papel del alumnolector, más pareciera que se trata de un sujeto abstracto, no situado, que aplica unas estrategias generales de lectura para lograr el uso eficiente de la lengua y, muy de paso, desarrollar una competencia literaria (concepto ambiguo por cierto). (Aguilar, 2016, p. 65)

Ante esta situación, Aguilar señala dos necesidades: 1. "La necesidad de enseñar literatura desde una teoría de la lectura literaria" (2016, p. 65), es decir desde una teoría específica y explícita, y no desde supuestos sin analizar, asumidos implícitamente, y 2 :

El desafío que debemos asumir a partir de estos y otros aportes es perfilar pedagógica y epistemológicamente un modelo integral y flexible de lectura literaria que considere tanto la esfera individual (encuentro íntimo entre el alumno-lector y el texto) como la esfera de socialización (puesta en común) de la lectura literaria. (p. 81) 
Como soluciones ante estos problemas y desafíos, han surgido propuestas como la educación literaria (Sánchez-Lara, 2020), que considera la enseñanza de la literatura de un modo específico, con características y necesidades distintas a las de la enseñanza de la lengua y de la expresión; que se constituye en un modelo que considera los aspectos mencionados anteriormente por Aguilar, y que está teniendo desarrollos teóricos y empíricos en diversos países de América Latina, tales como Chile (Sánchez-Lara, 2020), Colombia (Sanabria, 2018) y Argentina (Labarthe \& Herrera, 2016) ${ }^{1}$.

\section{Panorama específico: los programas de estudio y el canon literario escolar de educación media}

A través de un análisis de los programas de estudio de Lenguaje y Literatura, Aguilar (2013) muestra que los "sucesivos procesos de reforma y actualización curricular $[\ldots]$ no han implicado una transformación significativa del modelo didáctico-pedagógico instituido desde la reforma de 1968" (p. 178). De este modo, el autor señala entre los hallazgos de su estudio: 1. "las contradicciones entre el discurso de la reforma [de 2008], las acciones de las instancias reformadoras y la naturaleza que presenta el objeto reformado (en este caso los Progra- mas de Enseñanza del Lenguaje y la Literatura)", 2. "la falta de una consideración seria de las necesidades, intereses educativos y gustos lectores de los destinatarios de los planes y programas de enseñanza (estudiantes)" y 3. "la falta de participación de docentes en ejercicio [así como de otros especialistas] en la elaboración de las propuestas programáticas" (p. 183).

Una de las conclusiones del estudio es que "la enseñanza de la literatura [en El Salvador] se realiza con base en un enfoque historicista, europeizante y masculino"2 (p. 173). De este modo, Aguilar señala que

Algunas de las características fundamentales de este modelo que se mantienen a pesar de los distintos procesos de reforma y actualización son: (1) organización concéntrica de los contenidos[,] en el sentido de que, para el estudio de la literatura salvadoreña, debe pasarse por el estudio de la literatura universal, española, latinoamericana y centroamericana; (2) enseñanza de la literatura desde un enfoque lineal progresivo de estudio de la literatura, esto es, se parte del estudio de los clásicos grecorromanos para llegar al estudio de la literatura actual (modelo historicista) [enseñanza de la literatura según un modelo bistoricista]; (3) constitución de un canon literario escolar marcadamente europeo, masculino

1 En El Salvador ha existido la tendencia a contratar asesores y consultores extranjeros para las reformas educativas y los cambios curriculares, sin que dichas participaciones hayan implicado la capacitación de personal salvadoreño. Si aquí se señalan experiencias de renovación de la enseñanza de la literatura en América Latina, es para mostrar que en la región hay iniciativas con base pedagógica y científica (antes que con motivación comercial) y para sugerir el estudio de dichas experiencias por medio de sus correspondientes textos técnicos y académicos, y el establecimiento, de ser posible, de contacto con esos docentes e investigadores y sus instituciones y de acuerdos de cooperación.

2 Las cursivas son nuestras. 
y occidental y (4) tratamiento marginal de la literatura salvadoreña ${ }^{3}$. (2013, p. 178)

Acerca de los efectos educativos de un canon escolar con estas características, Aguilar observa que

[...] los mecanismos de canonización, a través del encubrimiento de la divergencia y de la invisibilización del Otro como interlocutor válido, estarían poniendo límites al desarrollo de capacidades críticas del alumnado. Constriñendo su libertad de interpretación por medio del control ideológico y de la defensa de determinados valores. (2012, pp. 59-60)

Nosotros agregamos que un canon de este tipo también pone límites al desarrollo de la inteligencia emocional (Gardner) del alumnado.

Al canon resultante de este modelo educativo, Aguilar lo denomina canon literario escolar pétreo, al cual define como

el conjunto de autores y obras literarias que han permanecido en el centro durante los distintos procesos de reforma y actualización de los programas de estudio, gracias a que responden satisfactoriamen- te al discurso oficial sobre la cultura[,] a la concepción dominante sobre los saberes escolares y al sistema de valores que se definen como prioridades de la enseñanza. (2013, p. 182)

Este canon "constituye un centro que se resiste a ser cuestionado desde el punto de vista de su representatividad, valor pedagógico y pertinencia cultural" (Aguilar, 2013, p. 182).

Además, Aguilar identifica en los programas de estudio "falencias relacionadas con su coherencia pedagógica y su pertinencia cultural" (2013, p. 187). El autor señala dos motivos principales:

[...] primero porque mezclan varios enfoques de enseñanza (gramática tradicional, estructuralista; pragmática literaria, enfoque comunicativo, pedagogía de medios) y los insertan dentro del modelo de competencias, el cual, a su vez, se cubre con el paraguas del constructivismo ${ }^{4}[\ldots]$. Segundo, porque este modelo soslaya el hecho de que el proceso de enseñanza está ligado a dinámicas concretas como las culturas escolares, las culturas comunitarias y los gustos lectores, por citar algunas de ellas. (Aguilar, 2013, p. 187)

3 Estas mismas características están presentes en los materiales educativos para Lenguaje y Literatura que se están implementando durante la pandemia de COVID-19. Por ejemplo, pueden comprobarse en las teleclases del área de Literatura para segundo año de bachillerato, en el canal de Youtube "Aprendamos en casa El Salvador". En: https://www.youtube.com/channel/UCt-1E5fqZI6YI2PBFApkPYA

4 El autor expone antecedentes y postulados básicos del constructivismo en Aguilar (2012, p. 27ss). Allí plantea que: “En el ámbito educativo, el constructivismo, aún en construcción, continúa siendo un marco de referencia valioso para analizar, problematizar y reflexionar sobre aspectos docentes, aprendizaje de los alumnos y cuestiones curriculares (Coll, 1991; Hernández, 1998), aunque ha sido objeto de varias lecturas e interpretaciones muchas veces erróneas en la educación latinoamericana" (p. 30). 
Aguilar también identifica una "ausencia de sustentación teórica, en los planes y programas, de la noción de pensamiento crítico" (2012, p. 333).

A estas falencias en los programas, hay que sumarle las grandes deficiencias de la puesta en práctica de los mismos, tal como lo constataron en su estudio Iraheta \& López (2014), quienes afirman que

[...] existe evidencia suficiente para sostener que los maestros y las maestras de Educación Media del sector público y algunos del sector privado [...] no ponen en práctica de manera adecuada el enfoque educativo por competencias en su trabajo de aula [...] se logró determinar por medio de la observación de campo y las entrevistas, que las formas de enseñar obedecen a un esquema acentuadamente bancario. (p. 72)

Por último, en el aspecto ideológico, Aguilar señala el carácter conservador del canon, tendiente a reproducir valores tradicionales en lugar de impulsar "una educación más democrática, abierta y con sentido de identidad nacional" (2013, pp. 185-186, 193-194).

\section{Recomendaciones}

\section{Una consideración previa: marcos socioculturales y pedagógicos del canon}

El canon literario escolar funciona en relación y condicionado por otros elementos, tanto socioculturales como educativos; por lo tanto, emprender cambios efectivos en el canon im- plica tener una perspectiva sistémica, holística, $\mathrm{y}$ así tener en cuenta las relaciones de este con dichos elementos.

En el caso salvadoreño, emprender cambios efectivos en el canon debería tener como marco acciones más amplias en el ámbito educativo, tales como: 1. diseñar y darle continuidad a un plan educativo de largo plazo, al menos para los siguientes 20 años (Picardo, 2012 y Álvarez, 2019); 2. diseñar un currículo escolar, pues El Salvador no tiene uno (Picardo, 10 julio 2020); 3. implementar de forma funcional un modelo educativo coherente y pertinente (Aguilar, 2013; Picardo \& Rovira, 2020 y Picardo, 10 julio 2020), pues actualmente los programas de estudio afirman que se desarrolla una educación con enfoque constructivista y basada en competencias, pero estudios, como Aguilar (2012 y 2013) e Iraheta \& López (2014), muestran que la enseñanza de la literatura sigue siendo bancaria, tradicional, basada en un modelo de enseñanza "abstracto y distante de las necesidades e intereses del alumnado, de la vida moderna y del mundo del trabajo" (Aguilar, 2013, p. 185); 4. articular la formación docente con dicho modelo educativo (Picardo, 10 de julio de 2020; Iraheta \& López, 2014); 5. hacer efectiva una dignificación de la carrera docente (Álvarez, 2019) y 6. asegurar recursos de lectura necesarios para la concreción del papel del canon literario escolar en el currículo, tales como fondos actualizados y atractivos para bibliotecas escolares y públicas, libros de texto e incluso libros del canon, y conexión de calidad a internet en las escuelas públicas (Picardo, 26 de abril de 2020). Son grandes retos, que es indispensable afrontar para poder desarrollar una educación de calidad. 
Estas acciones deberían realizarse orientadas por los grandes consensos nacionales e internacionales sobre para qué y cómo se debe educar. Entre dichos consensos se encuentran: los Fundamentos curriculares de la educación nacional (MINED, 1994), La educación encierra un tesoro (también conocido como el Informe Delors) (UNESCO, 1994), Los siete saberes necesarios para la educación del futuro (Edgar Morin, 1999), los Objetivos del Milenio (ONU) y los Objetivos de Desarrollo Sostenible (ONU). Es decir: no se necesita partir desde cero para emprender cambios educativos, pues disponemos de brújulas muy bien diseñadas, vigentes, y a partir de las cuales, se está trabajando en diversos países para lograr grandes objetivos comunes de la humanidad.

A continuación se exponen algunas recomendaciones para una renovación funcional del canon literario escolar de educación media en El Salvador.

\section{Modificar los objetivos y el modelo de enseñanza de la literatura}

El enfoque comunicativo y el enfoque basado en competencias demuestran limitaciones para una enseñanza significativa de la literatura (Aguilar 2012 y 2013; Sánchez Lara, 2020), por lo que es necesario cambiar los objetivos y el enfoque para su enseñanza.

Un enfoque que se está probando como más efectivo es el de la educación literaria (Sánchez-Lara, 2020), que entiende como objetivo principal de la enseñanza de la literatura el desarrollo de capacidades. Un ejemplo de capacidad relacionada a la lectura es la de leer por placer, ser capaz de leer por placer. Para motivar y enseñar a leer por placer se necesi$\tan$ marcos distintos a los de las competencias. (Y hay que notar que competencias y capacidades no son mutuamente excluyentes, sino complementarias). Así, retomando el ejemplo de la lectura por placer, ya se ha comprobado que el aprendizaje no es una operación meramente cognitiva, sino que tiene un componente emocional (Ábrego \& Picardo, 2019). La educación literaria atiende la necesidad de los estudiantes de involucrarse emocionalmente en sus aprendizajes, posibilitando aprendizajes que otros enfoques, como el basado en competencias, buscan en sus principios formales, pero que no alcanzan a través de sus métodos.

Sánchez-Lara (2020), en las conclusiones de su estudio, lo plantea de este modo:

La didáctica literaria y su peso teórico, debe cuestionarse, a la luz del tratamiento curricular que se ha establecido, la necesidad de generar, como la emergente educación literaria propone, una renovación respecto al sentido de "enseñar" literatura, relativizando las prácticas centrípetas de lectura y movilizándose hacia el desarrollo del sujeto, no del objeto, instalando discursos de acción pedagógica orientados desde un eidos [idea orientadora] crítico que suponga: movimientos centrífugos de lectura-escritura, fruición, interacción oral entre poéticas de aula, talleres de creación y comprensión de lo cultural por medio de significaciones que emerjan desde el estudiante hacia la problematización del canon y su verdad. (p.67) 
$\mathrm{Y}$ en el sentido de modificaciones en los métodos de enseñanza de la literatura, Sánchez-Lara (2020) señala entre los desafíos "acciones pedagógicas que den sustento empírico a la posibilidad de posicionar la escritura creativa en la escuela" (p. 67). Labarthe \& Herrera (2016) ya reportan una experiencia exitosa de taller de escritura creativa para potenciar el desarrollo humano en el aula. Y por nuestra parte, planteamos que es necesario integrar la escritura con la lectura en el aula, ya que:

«La lectura empodera, pero la escritura empodera más», [como] dice la educadora Silvia Castrillón. La escritura, en este sentido, es otra herramienta poderosa para contextualizar la enseñanza de la literatura y promover aprendizajes significativos y el desarrollo de capacidades, ya que promueve la creatividad, la capacidad de introspección, la construcción de significados y la creación de sentidos, el empoderamiento, la toma de decisiones y la autonomía (DeSalvo, 1999; Cameron, 2019), entre otras muchas capacidades. (Zetino, 2020, p. 61)

\section{Elaborar un conjunto de criterios básicos de selección de textos}

Tal como queda evidenciado en Aguilar (2013), cuyo autor entrevistó al equipo técnico encargado de la selección del canon literario escolar de bachillerato para la reforma educativa de 2008, una de las fa- lencias históricas de dicha selección es que se ha hecho con criterios no explícitos, ni para los equipos que lo han seleccionado ni para otros actores. Es decir: no existe un documento que exprese con qué criterios se seleccionan los textos que se leen en el bachillerato salvadoreño ${ }^{5}$. Un documento como este, basado en investigación y consultas series, puede ser una herramienta fundamental para la renovación del canon y para una actualización continua efectiva de este.

Algunos de los criterios que se deberían tomar en cuenta son:

\section{a. Las necesidades, intereses y gustos lectores de los estudiantes}

Aguilar (2013) ha señalado "la falta de una consideración seria de las necesidades, intereses educativos y gustos lectores de los destinatarios de los planes y programas de enseñanza (estudiantes)" (p. 183). Nosotros consideramos que la primera necesidad de los estudiantes que debe atender el canon literario escolar es la motivación, tanto en su selección de textos como en las prácticas educativas con estos. Sobre la motivación a la lectura hemos tratado en Zetino (2014).

Muy relacionada con la motivación, una necesidad acuciante en el bachillerato salvadoreño es la inclusión de literatura juvenil en los textos del canon. La ONG educativa

5 Y acerca de la selección de textos en sí, esta se encuentra distribuida a lo largo de los programas de estudio (MINED, 2008).

6 La cursiva es nuestra. 
ConTextos (contextos.org) responde a esta necesidad con la selección de la biblioteca con que dota a las instituciones que interviene, que incluye textos del canon oficial y también textos juveniles. También se puede trabajar con la especificidad de la recomendación de Oyarzún (2009, p. 233): "Incluir elementos de la cultura juvenil en los textos escolares".

Y una tercera necesidad propia de la realidad social salvadoreña es la de la promoción de una cultura de paz. El canon de la biblioteca de ConTextos y sus prácticas en instituciones educativas a partir de él son un caso de éxito de promoción de convivencia y de desarrollo de resiliencia a partir de la lectura y la escritura.

\section{b. Los objetivos pedagógicos de los textos incluidos}

¿Para qué se utilizarán los textos que se incluyen en el canon literario escolar? ¿Cuáles capacidades y competencias se desarrollarán a través de su uso escolar? Estos objetivos también deben ser explícitos, y pueden llevar a una reformulación del canon en diversos sentidos.

Primero, como se señaló en la revisión de la literatura, el canon literario escolar de educación media es historicista y lineal (Aguilar, 2012 y 2013): se realiza como un estudio de la historia de la literatura y trata de ir desde la antigüedad griega hasta literatura salvadoreña contemporánea. Esto, como puede verse, pone el uso educativo de la literatura en función de la historia de la literatura; el rum- bo principal de los aprendizajes sería el que pueda derivarse de ese recorrido histórico.

Ahora, al partir desde otro punto, de las preguntas "¿qué buscamos enseñar?”, “¿por qué enseñar esto?” y “¿para qué enseñar esto?”, las posibilidades pueden ampliarse muchísimo, pues el uso educativo de la literatura se hará en función de los aprendizajes buscados.

Una concreción de este planteamiento puede encontrarse en la antología (en inglés: $r$ ader) A Sense of Wonder: Reading and Writing Through Literature (Preston, 2002). Este libro de texto está diseñado para enseñar inglés como segunda lengua, y está dividido en seis capítulos temáticos que exploran aspectos: comida, amor, ropa, crecer, trabajo, vida y muerte. Cada uno de estos aspectos es abordado a través de textos de diversos géneros literarios (poesía, cuento, teatro y ensayo), a partir de los cuales se desarrollan diversas actividades de discusión, interpretación y creación. Se agrega que los textos son de autores de diversos orígenes nacionales y culturales, y que los géneros de los textos no tienen un orden fijo (por ejemplo: no siguen todos la secuencia poesía-cuento-teatro-ensayo), sino que van variando, e incluso hay capítulos que no los contienen todos.

Pensar en cambios como estos puede remitir a debates sobre la conformación del canon literario escolar: cadmitirá libros que no son clásicos?, ¿admitirá muchos textos que no son clásicos?, ¿dejará de tener clásicos?, ¿dejará de representar "los grandes valores de la civilización occidental?, etc. Aguilar (2012, pp. 53-68), desarrolla una exposi- 
ción detallada de las relaciones entre el canon literario escolar y la enseñanza de la literatura, problematizando aspectos como la relación entre la noción de canon literario y valor (cultural); los debates sobre el canon literario de las décadas recientes en Estados Unidos, que han llegado a contraponer, de modo a veces conflictivo, "unidad cultural" y multiculturalidad, lo clásico y lo contemporáneo, la representación de la cultura hegemónica y la de minorías culturales, etc.; e implicaciones de la teoría del canon literario en la enseñanza de la literatura. Nuestra postura en este punto es que un marco de referencia como el de Aguilar (2012) debería poder fundamentar una discusión para la apertura, la ampliación, la renovación de nuestro canon literario escolar de educación media (y también el de tercer ciclo, estructurado según principios semejantes), al facilitar una comprensión mayor sobre el canon literario en su instrumentación escolar.

Para concluir este punto, sugerimos pensar en un canon funcional para la educación como ideal. El criterio de los objetivos educativos de este canon será el que determine los textos que lo integren.

\section{c. La igualdad de género en la selección de los textos}

Otro criterio de selección de textos debe ser el balance entre autores hombres y mujeres. Aguilar (2012 y 2013) ya ha señalado el canon literario escolar de educación media es masculinizante: de 51 autores que contiene, sólo uno es una mujer (Aguilar,
2013, p. 180). Los motivos de tomar en serio este criterio son los de la formación de visión de mundo y de valores y el desarrollo emocional y también de pensamiento crítico. Aguilar (2012, p. 198) señala que “ [...] se trata de una literatura en la que se acentúa de manera muy particular la presencia de los estereotipos sexistas y las desiguales relaciones de poder-dominio entre hombres y mujeres".

No estamos sugiriendo una selección de textos que excluya a toda costa estereotipos o desigualdades: esos son hechos de la realidad sociohistórica y sociocultural, que han sido y siguen siendo expresados en el arte, acerca de los cuales se debe enseñar a tener un pensamiento crítico. A lo que apuntamos con el criterio de balance de autores de ambos sexos es a la promoción de la igualdad de género; a un conocimiento más amplio y más completo de la naturaleza humana, la sociedad y la historia; al reconocimiento del otro como interlocutor válido; a la exposición a y la consideración de puntos de vista diversos y a la formación de una sensibilidad más funcional, es decir un desarrollo más pleno de la inteligencia emocional (Gardner).

\section{d. La representación de la literatura salvadoreña}

Aguilar (2013) también ha señalado la escasa inclusión de literatura salvadoreña en el canon literario escolar de educación media, y es muy conocido el hecho de que podría no ser estudiada, pues el tiempo del año tal vez no alcance para llegar hasta ese punto 
del programa. Esto tiene gran influencia en la alfabetización cultural, que incluye el desarrollo de la identidad cultural. Una estructuración del canon según el criterio de objetivos pedagógicos de los textos ayudaría a un conocimiento mayor de la literatura salvadoreña, al no dejarla al final de una secuencia, sino al distribuir textos de ella en un programa que podría ser temático, como el texto de Preston (2002) que citamos en el apartado b.

\section{Crear un equipo multidisciplinario para elaborar un nuevo canon literario}

En cuanto a la conformación del equipo, este debería constar por lo de: especialistas de Letras; educadores: curriculistas, docentes e investigadores educativos; posiblemente también escritores; y tener un balance generacional: tanto miembros experimentados como jóvenes. Esta elaboración debe ser lo más sistemática posible, hacerse a través de unos criterios definidos y explícitos para el equipo (tales como los que estamos proponiendo), y ser validada con grupos pilotos de estudiantes, docentes de escuelas y de profesorado y estudiantes de profesorado.

\section{Capitalizar experiencias de renovación del canon literario escolar en El Salvador}

En el país contamos con una experiencia exitosa y sostenida a lo largo del tiempo: la de la ONG educativa ConTextos. Su estra- tegia de selección de libros debe ser estudiada como caso de buena práctica de educación literaria, además de la red de relaciones que la ONG crea para garantizar la continuidad de su propuesta: formación docente continua; estrategias para el desarrollo del pensamiento crítico, la convivencia y la resiliencia a través de lectura y la escritura creativa; donación de biblioteca a la escuela intervenida; capacitación a bibliotecarios (que sobre todo son estudiantes) y seguimiento de dichas acciones.

\section{Crear y darle continuidad a un observatorio de estudios y buenas prácticas de enseñanza de la literatura, tanto en el país como a nivel internacional}

Como ejemplos de esto se pueden mencionar: el caso del sistema de enseñanza de la literatura de la ONG ConTextos; la Revista OCNOS (Universidad de Castilla-La Mancha), especializada en estudios sobre lectura; el Centro de Estudios en Educación y Aprendizaje Basado en la Comunidad (Universidad Católica Silva Henríquez, Chile); y Lukuinto (El Gozo de Leer), el programa de tres años que el Ministerio de Educación y Cultura de Finlandia implementó con el objetivo de "develop comprehensive reading skills in children and young people and to promote the joy of reading through cooperation between school, library and home" (University of Oulu y Ministerio de Educación y Cultura de Finlandia, s.f.). Se elaboró un manual de dicho programa 
(University of Oulu, 2015) ${ }^{7}$. Además, en El Salvador, la Universidad Francisco Gavidia ha realizado durante dos años el Encuentro Universitario "La lectura y cambio generacional", de relevancia para la reflexión y la actualización sobre la lectura en el contexto escolar.

Cerramos este apartado señalando que: en la renovación del canon literario escolar, lo más importante no es la lista de libros de que lo integran, sino cómo se llega a esa lista: el conjunto de criterios y procedimientos para elaborarla, y cómo se la utiliza: el conjunto de prácticas educativas a partir y alrededor del canon.

\section{El canon literario escolar de educa- ción media durante la pandemia de COVID-19}

Antes de concluir este artículo, es necesario aún exponer algunas consideraciones para la optimización del canon de este nivel educativo durante la pandemia de COVID-19.

Lo primero que deseamos señalar es que la emergencia no es un motivo para suspender las investigaciones y las acciones para el mejoramiento de este elemento educativo, sino que, por el contrario, la necesidad urgente de posibilitar aprendizajes significativos en una educación a distancia, hace que la inves- tigación y las acciones en esta área tengan pleno sentido, pues buscan la mejor calidad educativa posible para los estudiantes del sector público.

Y al tocar el punto de la educación a distancia, no dejamos de tener en cuenta las grandes dificultades que esta implica en El Salvador (Picardo, 26 abril 2020 y 29 julio 2020) y que debido a la pobreza y la falta de los recursos adecuados, es un número limitado de estudiante el que la está realizando. Sin embargo, como ya lo afirmamos, aún con esas dificultades es necesario garantizar por todos los medios la calidad educativa, tanto para la educación del presente como para la del futuro. Y uno de los medios para hacer esto, un elemento educativo de enorme influencia, es el canon literario escolar. De allí la importancia de seguir trabajando en él.

Las recomendaciones específicas que hacemos para la optimización del canon de educación media en la educación a distancia son:

\section{Reducir el carácter historicista, eurocéntrico y masculinizante de los textos utilizados para la educación a distancia}

Como ya se señaló en la revisión de la literatura, las guías de estudio y las teleclases de literatura continúan teniendo estas carac-

7 Argentina tiene un acuerdo con Finlandia para la cooperación educativa. "Tenemos que tener la humildad de observar las buenas prácticas de otros países. No para copiarlas literalmente, sino para ver si algunas de ellas pueden adaptarse a nuestra realidad concreta”, afirmó Alejandro Finocchiaro, ministro de educación argentino de esa época, en ocasión de la firma del acuerdo. (La Nación, 2 de noviembre de 2017). En: https://www.lanacion.com.ar/economia/finlandia-asesorara-a-la-argentina-en-el-diseno-educativo-y-la-formacion-docente-nid2078713). 
terísticas. Las recomendaciones que damos en el apartado anterior pueden servir para orientar cambios en estos aspectos.

\section{Propiciar las experiencias literarias}

“Construir 'experiencia literaria' [es] uno de los objetivos más importantes de todo docente de Letras", plantea, en consonancia con el constructivismo, Bueno (2013, p. 69). Tres formas posibles de hacerlo en la educación a distancia son: la lectura en voz alta para los estudiantes, las lecturas complementarias atrayentes y las prácticas de escritura creativa.

\section{a. Leerles en voz alta a los estudiantes}

El profesor de literatura e investigador colombiano Emilio Calderón Reyes plantea alcances de leerles literatura en voz alta a los estudiantes:

Al leer en voz alta se le da una vida y corporalidad al texto. Aspirar a replicar esa transformación es a lo que deben apuntar gran parte de los esfuerzos de quien enseña literatura, sin importar la edad ni el nivel educativo en el que trabaje. Un profesor de literatura que les lea a sus alumnos y promueva la lectura en voz alta fomenta relaciones más directas y vitales entre el texto y las personas ${ }^{8}$. Además, al hacerlo, pone el énfasis del aprendizaje en la imaginación y la experiencia. Por más de que toda el aula permanezca en silencio, no hay que confundir lo que sucede [en el caso específico de leer en voz alta] con pasividad, puesto [que] la apreciación estética y el deleite son momentos de recogimiento. (Calderón, 2020, p. 37)

Calderón ilustra su planeamiento con un fragmento de Como una novela, de Daniel Pennac, libro referente sobre motivación a la lectura:

Vale la pena ver cómo el escritor [Pennac] recuerda a su maestro favorito de la escuela: "A través de su voz descubríamos de pronto que todo eso había sido escrito para nosotros. Este descubrimiento venía a ocurrir después de una interminable escolaridad en la que la enseñanza de la literatura nos había mantenido a distancia respetuosa de los libros" (Pennac, 2002, p. 86). (Calderón, 2020, p. 37)

De este modo, leer en voz alta en las teleclases (que sería el aspecto más asequible de la educación a distancia, en comparación con las guías de estudio, que requieren de internet), textos breves o fragmentos para los estudiantes, es decir leerles con intencionalidad, sería un modo de construir experiencia literaria o experiencia de la literatura. Esta es una limitación del enfoque basado en competencias: una competencia se puede plantear que el estudiante lea con entusiasmo textos literarios, pero muchísimas veces no hay nada en el contexto de ese acto de lectura (ni el texto en sí mismo, ni acciones de motivación a la lectura por parte de los profesores, que a su vez en muchísimos casos no poseen el trasfondo necesario para motivar a la lectura) que propicie el entusiasmo. 
Además de los alcances planteados por Calderón, la lectura en voz alta para los estudiantes puede ser una forma de acompañamiento, de estar con ellos aun en la distancia, lo cual sería un factor de humanización de la enseñanzaaprendizaje.

\section{b. Sugerir lecturas complementarias motivadoras, atrayentes, disponibles en internet}

Otra forma de propiciar experiencias literarias es la sugerencia de lecturas complementarias motivadoras, atrayentes. Subrayamos estas últimas palabras, porque nadie pasará por alto que el texto que se está utilizando en una clase determinada en muchos casos no es atrayente para el estudiante, debido a diversos motivos, tales como la ausencia del trasfondo cultural y del conocimiento lingüístico necesarios para la comprensión del texto.

En la modalidad de educación a distancia, pueden recomendarse sitios web con contenidos de lectura y presentación atrayente para los estudiantes. Ejemplos: el sitio amediavoz. com, que es una antología de poesía de todo el mundo y toda la historia. Se pueden recomendar algunas selecciones del sitio, y ya luego los estudiantes, si este ha sido de su interés, pueden seguir explorándolo por cuenta propia. Otro ejemplo son las revistas literarias, que forman parte de la alfabetización cultural a través de la literatura. Del mismo modo que con un sitio web, se pueden seleccionar publicaciones específicas (y la ventaja con una revista es que suele publicar todos los géneros literarios) y luego los estudiantes se quedarán frecuentándola según les atraiga. Una revista salvadoreña de gran calidad que se puede utilizar para este propósito es La Zebra. Sería también una forma de poner a los estudiantes con autores y tendencias literarias contemporáneos, tanto nacionales como regionales e internacionales.

Por último, pero no menos importante, la Biblioteca Virtual de Literatura Salvadoreña del MINED 9 es un recurso con un potencial enorme para animar a la lectura con lecturas complementarias. En mayo pasado, el MINED habilitó esta sección de su sitio con tres libros completos de Roque Dalton en pdf, lo cual fue una acción muy positiva. Sin embargo, no se le ha dado continuidad a la biblioteca. Y esta continuidad podría hacerse de formas muy sencillas, como la publicación de pequeñas muestras o de antologías mínimas de poetas salvadoreños, digamos un documento con 10 poemas, con una edición atrayente. Esto puede motivar a la lectura, da a conocer a autores poco conocidos o aspectos poco conocidos de ellos, y también sería una forma de promover la igualdad de género, al publicar a más escritoras.

\section{c. Promover prácticas de escritura creativa}

La escritura utilizada de forma creativa (que no necesariamente es sinónimo de "artística") es un factor poderoso de promoción de aprendizajes significativos. Para ampliar este punto véase el literal 1 del apartado anterior, "Recomendaciones". 


\section{AKADEMOS \\ 3. Contextualizar los textos literarios utilizados}

Se pueden orientar los textos del programa o elegir otros de modo que el trabajo con ellos oriente a la comprensión y a la creación de sentidos ante las dificultades que conlleva la pandemia. Se puede trabajar con textos con situaciones en las que los personajes superan dificultades, resuelven problemas (tanto individualmente como junto a otros), encuentran esperanza, superan prejuicios, se organizan y trabajan junto a otros por objetivos comunes, son solidarios, leen o escriben y así aprenden o encuentran algo valioso, etc.

\section{Adecuar el lenguaje técnico}

La observación de las teleclases de literatura muestra que en ocasiones se está utilizando un lenguaje demasiado técnico, que debe ser adecuado para posibilitar la comprensión de los estudiantes. En palabras de una exalumna de este autor, que este año cursa segundo año de bachillerato: "en algunos momentos el lenguaje que los maestros usan (no solo en esta materia, si no, [sic] en todas) es muy técnico y por momentos es difícil llevar la continuidad de la idea" (comunicación personal, 10 de junio de 2020).

\section{Conclusiones}

La revisión de la literatura sobre el canon literario escolar de educación media en El Salvador muestra que hay muy pocos estudios en este tema, siendo los fundamentales los tres realizados por Aguilar (2012, 2013 y 2016). Este investigador, con base en un estudio empírico, asegura que "La enseñanza de la litera- tura en la escuela secundaria [salvadoreña] necesita ser repensada" (2012, p. 329). Entre sus aportes, se encuentran: 1 . el señalamiento de falencias teóricas y limitaciones pedagógicas en el modelo de enseñanza de la Didáctica de la Lengua y la Literatura (DLL), en el enfoque comunicativo de esta, y en el enfoque basado en competencias aplicado a la enseñanza de la literatura; 2. la identificación de falencias de coherencia pedagógica y de pertinencia cultural en los programas de estudio de Lenguaje y Literatura; 3. la caracterización del modelo de enseñanza de la literatura en El Salvador y del canon literario escolar de educación media como historicistas, europeizantes, masculinos y conservadores. Otro estudio empírico (Iraheta \& López, 2014) constata que los docentes no están formados para desarrollar un proceso de enseñanza-aprendizaje en literatura enfocado en el desarrollo de competencias, sino que la enseñanza en este campo sigue un esquema bancario.

Ante esta situación, Aguilar (2016) señala dos necesidades: enseñar literatura a partir de una teoría definida y explícita de la lectura literaria y "perfilar pedagógica y epistemológicamente un modelo integral y flexible de lectura literaria que considere tanto la esfera individual (encuentro íntimo entre el alumno-lector y el texto) como la esfera de socialización (puesta en común) de la lectura literaria” (p. 81).

Como solución ante estos problemas y desafíos, se plantean propuestas como la educación literaria, que considera la enseñanza de la literatura de un modo específico; incluye el desarrollo de capacidades, otorgándoles un lugar central, y está teniendo desarrollos teóricos y empíricos en diversos países de América Latina. 
A partir de este marco conceptual se formulan recomendaciones para una renovación funcional del canon literario escolar de educación media: 1. Modificar los objetivos y el modelo de enseñanza de la literatura, 2. Elaborar un conjunto de criterios básicos de selección de textos, 3. Crear un equipo multidisciplinario para elaborar un nuevo canon literario, 4. Capitalizar experiencias de renovación del canon literario escolar en El Salvador, y 5. Crear y darle continuidad a un observatorio de estudios y buenas prácticas de enseñanza de la literatura, tanto en el país como a nivel internacional. Un caso exitoso y de amplio impacto de educación literaria y de renovación del canon literario escolar en El Salvador es de la ONG Contextos, el cual se sugiere conocer y estudiar, y capitalizar su sistema y sus logros.

Asimismo, se exponen algunas consideraciones para la optimización del canon de educación media en la educación pública durante la pandemia de COVID-19. Estas son: 1. Reducir el carácter historicista, eurocéntrico y masculinizante de los textos utilizados para la educación a distancia; 2. Propiciar las experiencias literarias, por medio de acciones como: a. Leerles en voz alta a los estudiantes, b. Sugerir lecturas complementarias mo- tivadoras, atrayentes, disponibles en internet (parte de lo cual puede hacerse a través de la biblioteca virtual del MINED), y c. Promover prácticas de escritura creativa; 3. Contextualizar los textos literarios utilizados, y 4. Adecuar el lenguaje técnico empleado en las teleclases.

Se estima que, en general, el marco teórico y las recomendaciones de este artículo son extrapolables a tercer ciclo de educación básica.

Por último, es esencial tener en mente que en la renovación del canon literario escolar, lo más importante no es la lista de libros de que lo integran, sino cómo se llega a esa lista: el conjunto de criterios y procedimientos para elaborarla, y cómo se la utiliza: el conjunto de prácticas educativas a partir y alrededor del canon.

Y es urgente emprender la renovación del canon literario escolar y de sus prácticas ahora, para garantizarles una educación de calidad a los estudiantes de educación media. Para hacerles posible una formación integral, humana y humanizadora, que les permita afrontar la crisis por la pandemia de COVID-19, y gozar de la cultura, que es patrimonio suyo, expresarse, crear y afrontar de manera constructiva los desafíos de su vida futura. 
AKADEMOS Enero-Junio 2020, n. ${ }^{\circ} 34$, ISSN: 1995-4743

\section{Referencias}

1 Aguilar Ciciliano, M. (2012). El papel de la literatura en el desarrollo del pensamiento crítico de las y los estudiantes de educación media: el caso salvadoreño. (Tesis de doctorado). Universidad de Costa Rica.

2 Aguilar Ciciliano, M. (2013). Canon literario escolar y enseñanza de la literatura en la educación media: Un análisis crítico de los programas de enseñanza secundaria en El Salvador. Educare, 17(3), 173-198. DOI: https://doi.org/10.15359/ree.17-3.9

3 Aguilar Ciciliano, M. (2016). El concepto de la lectura literaria en la pedagogía actual de la literatura. $L a$ Universidad, 31. 63-85. En: http://revistas.ues.edu.sv/ index.php/launiversidad/article/view/765

4 Álvarez, F. (2019). Análisis prospectivo del sistema educativo salvadoreño. (Documento en pdf). San Salvador: Universidad Francisco Gavidia. En: http://blogufg. com/wp-content/uploads/2019/05/Analisis_del_Sistema_Educativo_de_El_Salvador_UFG_Francisco_Alvarez_Abril_2019.pdf

5 Bueno, M. (2013). ¿Por qué enseñar literatura? abehache, 4(1), 53-72. En: http://www.hispanistas.org.br/ arquivos/revistas/abehache4.pdf\#page $=52$

6 Calderón Reyes, E. Volver al aula como literato: educación literaria y constructivismo. En: Gómez Lozano, M. T. \& Vinasco Castaneda, C. (Comp.). (2020). La voz del estudiante en la educación superior. Bogotá: Ediciones Uniandes. Págs. 33-41.

7 Iraheta, I. M. \& López, I. E. (2014). Análisis del enfoque educativo por competencias aplicado en la enseñanza de Lenguaje y Literatura en el Programa de Estudio de Educación Media en El Salvador. Universidad de El Salvador, Facultad Multidisciplinaria de Occidente. (Tesis de Licenciatura). En: http://ri.ues.edu.sv/id/ eprint/13077

8 Labarthe, J. T. \& Herrera Vásquez, L. (2016). Potenciando la creatividad humana: taller de escritura creativa. Papeles de Trabajo, 31, 19-37. En: http://hdl.handle. net/2133/12783

9 MINED. (1994). Fundamentos curriculares de la educa- ción nacional. (Documento en pdf). En: https://webquery.ujmd.edu.sv/siab/bvirtual/BIBLIOTECA $\% 20$ VIRTUAL/LIBROS/F/ADMF0000411.pdf

10 MINED. (2008). Programas de estudio. Lenguaje y Literatura. Educación Media. (Documento en pdf). En: https://www.mined.gob.sv/descargas/send/866programa-de-estudio-de-educacion-media/5502lenguaje-educ-media-0.html

11 Ministerio de Educación y Cultura de Finlandia. (s.f.). Joy of Reading. (Página web del programa educativo del mismo nombre). En: http://lukuinto.fi/joy-of-reading.html

12 Oyarzún, L. (2009). Requerimientos técnicos y criterios de selección de textos escolares de Lenguaje y Comunicación: logros y desafíos. Seminario Internaciones "Textos Escolares de Lenguaje y Comunicación". Santiago de Chile: Ministerio de Educación, 2010. (Documento PDF). Págs. 223-234. En: https:// docplayer.es/8529287-Textos-escolares-de-lenguajey-comunicacion.html

13 Picardo Joao, O. (2012). Políticas públicas educativas: Ensayos para el debate. Antiguo Cuscatlán, El Salvador: Centro de Investigaciones en Ciencias y Humanidades $(\mathrm{CICH})$, Universidad Dr. José Matías Delgado.

14 Picardo Joao, O. (26 abril 2020). COVID 19 y Educación: Problemas y desafíos. El Diario de Hoy (versión digital). Consultado el 4 de mayo de 2020. En: https:// www.elsalvador.com/opinion/editoriales/educacionvirtual/709079/2020/

15 Picardo Joao, O. (22 junio 2020). La nueva normalidad, la nueva escuela. El Diario de Hoy (versión digital). Consultado el 23 de junio de 2020. En: https:// www.elsalvador.com/opinion/editoriales/educacion/726381/2020/

16 Picardo Joao, O. (26 julio 2020). ¿El fin de la educación pública...? . El Diario de Hoy (versión digital). Consultado el 26 de julio de 2020. En: https://www. elsalvador.com/opinion/editoriales/pandemia-educacion/736661/2020/ 
17 Picardo Joao, O. \& Ábrego, A. M. (2019). La curva del aprendizaje: Un enfoque neuroevolutivo. San Salvador: Instituto de Ciencia, Tecnología e Innovación (ICTI), Universidad Francisco Gavidia.

18 Picardo Joao, O. \& Rovira, C. (2020). Educación después del COVID-19. ¿Qué podemos aprender de esta experiencia? (Foro). Instituto de Ciencia, Tecnología e Innovación (ICTI), Universidad Francisco Gavidia. (24 junio 2020). (Parte I del Foro Pedagógico “Educación y COVID-19: Lecciones y desafíos para la educación luego de la pandemia). En: https://www.facebook. com/Disruptiva.Media/videos/622378761992267/ $? \mathrm{v}=622378761992267$

19 Preston, B. (2002). A Sense of Wonder: Reading and Writing Through Literature. New Jersey: Pearson Education.

20 Sanabria Ardila, L. M. (2018). Transformación del canon literario formativo para contribuir en el desarrollo de la competencia lectora y el deleite literario desde la novela colombiana femenina del siglo XXI. Ibagué, Colombia: Universidad del Tolima. (Tesis de maestría). En: http://repository.ut.edu.co/handle/001/2599

21 Sánchez-Lara, R. (2020). ¿Didáctica literaria o educación literaria? Una discusión desde el eidos curricular en las actividades de aprendizaje del libro de texto de segundo año medio. Revista de Educación y Desarrollo, 52, 59-68. En: http://www. cucs.udg.mx/revistas/edu_desarrollo/anteriores/52/52_SanchezLara.pdf

22 University of Oulu. (2015). The Joy of Reading Handbook. Improving reading motivation and multiriteracy through collaboration between schools and libraries. (Documento en pdf). En: http://lukuinto.fi/media/ materiaalit/joy-of-reading-handbook.pdf

23 Zetino, M. (2014). Por qué no leemos. Factores de desanimación a la lectura y propuestas para la animación. Akademos, 23(3), pp. 9-17. En internet: https://www. academia.edu/40263043/Por_que_no_leemos._Factores_de_desanimaci\%C3\%B3n_a_la_lectura_y_propuestas_para_la_animaci\%C3\%B3n

24 Zetino, M. (2020). Leer literatura: potenciales y propuestas ante la pandemia de COVID-19. COVID-19: Una mirada interdisciplinaria a la pandemia. (Boletín especial). Centro de Investigaciones en Ciencias y Humanidades, Universidad Dr. José Matías Delgado. Págs. 55-64. En: http://hdl.handle. net/10972/4149 
